\title{
The Benefit of Education and Appropriate Antibiotics Use to Reduce Multidrug-Resistant Acinetobacter baumanii in a University-Affiliated Intensive Care Unit in Indonesia
}

\author{
Cucunawangsih", Leni Lukman', R. Sariwijaya1, P. Wibowo², V. Sungono ${ }^{3}$ \\ ${ }^{1}$ Department of Microbiology, Faculty of Medicine, University of Pelita Harapan, Tangerang, Indonesia \\ ${ }^{2}$ Intensive Care Unit, Siloam General Hospital, Tangerang, Indonesia \\ ${ }^{3}$ Department of Public Health, Faculty of Medicine, University of Pelita Harapan, Tangerang, Indonesia \\ Email: cucunawangsih.fk@uph.edu
}

Received 22 September 2015; accepted 22 November 2015; published 25 November 2015

Copyright (C) 2015 by authors and Scientific Research Publishing Inc.

This work is licensed under the Creative Commons Attribution International License (CC BY).

http://creativecommons.org/licenses/by/4.0/

(c) (i) Open Access

\begin{abstract}
Acinetobacter baumanii is an opportunistic pathogen known to cause hospital acquired infection presenting with varying clinical feature from simply to much more severe manifestation. More importantly, widely improper and overuse of antibiotics consumption have caused an endemic of multidrug-resistant-Acinetobacter baumanii leading to prolonged hospital stay and poorer prognosis for intensive care patients. A descriptive study of pre- and post-education was conducted at an intensive care setting in Indonesia. The microbiology data were collected to evaluate the benefit of education on hand hygiene and management of antibiotic use to reduce the number of MDRAcinetobacter baumanii infection. Based on the result of previous local susceptibility patterns, Cefoperazone/Sulbactam and Amikacin are favored as the empirical therapy. Chi-square analysis shows the significant reduction of Acinetobacter baumanii cases from $70.8 \%(17 / 24)$ to $38 \%(3 / 8)$ with $P$-value 0.006 . Similarly, the susceptibility rate significantly increased, from $21 \%$ to $100 \%$ to Amikacin; from 5\% to $89 \%$ to Piperacillin/Tazobactam, and from $42 \%$ to $89 \%$ to Meropenem. Education improving around hand hygiene, appropriate antibiotic prescribing following local hospital guidelines and the result of antibiotic susceptibility has been shown to reduce the transmission of MDR-Acinetobacter baumanii in the intensive care in our unit within this Indonesian hospital.
\end{abstract}

\section{Keywords}

Acinetobacter baumanii, Hand Hygiene, Antibiotic Prescribing, Intensive Care

How to cite this paper: Cucunawangsih, et al. (2015) The Benefit of Education and Appropriate Antibiotics Use to Reduce Multidrug-Resistant Acinetobacter baumanii in a University-Affiliated Intensive Care Unit in Indonesia. Open Journal of Medical Microbiology, 5, 163-168. http://dx.doi.org/10.4236/ojmm.2015.54020 


\section{Introduction}

Acinetobacter spp. is non-fermentative gram-negative coccobacillus which has been increasing recently in accordance with rising cases of multi-drug resistant organisms [1] [2]. The most important characteristics of Acinetobacter baumanii are its high survival ability and resistance to wide range of antimicrobials [3] [4]. The clinical feature is ranging from colonization to severe manifestation [1] [2]. This condition is often met in hospitalized patients with severe illness and impaired host defense that needs medical support. The major manifestation of the infection varies from pneumonia, soft tissue infection, urinary tract infection, meningitis, and bacteraemia [5] [6].

Acinetobacter baumanii crude death rate is comparable to other gram-negative bacteria (28\% to 32\%) [1] [5], and the epidemic of this bacteria has become a global threat [7]. One of the primary causes of this phenomenon is uncontrolled use of antimicrobials in both of the community and hospital [8] [9]. Furthermore, the lack of resources, inadequately trained healthcare workers on education around hand hygiene, good knowledge of antibiotics and microbiology specimens handling supported this problem especially in developing countries [10]. MDR-Acinetobacter baumanii can lead to the poorer prognosis of the patients in intensive care and hospital infection control has to be done to prevent transmission among patients and healthcare workers. Therefore, strategy on education and appropriate antibiotics use is essential in order to reduce health care-associated infections. In this study, we sought to evaluate the benefit of hand hygiene promotion on health care worker and appropriate antibiotic use in reducing the number of MDR-Acinetobacter baumanii infection among intensive care patients.

\section{Materials and Methods}

This study was approved by Mochtar Riady Institute for Nanotechnology Ethics Committee (042/MRIN-EC/11/ 2013). This study was conducted in intensive care unit of a University of Pelita Harapan-affiliated hospital located in Tangerang, Indonesia and consisted of two steps. The first was data of antibiotic susceptibility patterns from June 2012-June 2013. The second step was initiated in September 2013 in the form of education on microbiology specimen handling, hand hygiene and socialization of appropriate antibiotic use to health workers and clinicians. Second data gathering on antibiotic susceptibility patterns was held in October 2013-March 2014. Discussion with clinicians, clinical microbiologists, and clinical pharmacologist with the help of local antibiotic guideline was done to select an appropriate antibiotic therapy.

Clinical samples were inoculated directly onto Columbia blood agar base ${ }^{\circledR}\left(\right.$ Difco $^{\mathrm{TM}}$ ) with sheep blood agar $5 \%$ and Mac Conkey agar (Difco $\left.{ }^{\mathrm{TM}}\right)$. Blood samples were cultured using a BD BACTEC ${ }^{\mathrm{TM}}$ blood culture system. All positive blood cultures were sub-cultured onto the same agar. Acinetobacter baumanii identification and antimicrobial susceptibility testing was completed using an automated method from VITEX-2 Compact ${ }^{\circledR}$ (Biomérieux, France) and CLSI (Clinical Laboratory and Standards Institute) protocols [11]. Escherichia coli ATCC $^{\circledR}$ 25,922 and Pseudomonas aeruginosa ATCC $^{\circledR} 27,853$ were used as control isolate for susceptibility testing.

Isolate distribution data and antibiotic sensitivity were compiled using WHOnet software (WHOnet 5.4) and analyzed with STATA version 10.0. Statistical significance was calculated by using chi-squared analysis. A $P$-value $<0.05$ was defined to be statistically significant.

\section{Results}

Table 1 showed the total number of culture examination during pre-education and post-education. Sputum was the most common cultured specimen in the period of June 2012-June 2013 with total percentage of $84.5 \%$ (33/39), followed by blood 13\% (3/39) and bronchial secretion 2.5\% (1/39). In June 2012-June 2013, a total of 23/39 (60\%) patients were suffering from bacterial infection consist of Acinetobacter baumanii 17/24 (70.8\%), Acinetobacter iwolfii 1/24 (4.2\%), Pseudomonas aeruginosa 2/24 (8.3\%), and 16.7\% gram positive bacteria isolates (Figure 1).

In post-education periode, Pseudomonas aeruginosa 4/13 (30.7\%) was the most common organism, followed byAcinetobacter baumnaii 3/13 (23\%). Staphylococcus aureus and Coagulase negative staphylococci (CoNS) isolates consisted of Staphylococcus capitis1/13 (7.7\%), Staphylococcus hominis1/13 (7.7\%), Staphylococcus hemoliticus1/13 (7.7\%), Staphylococcus saprophyticus1/13 (7.7\%) were the highest isolates found in October 2013 to March 2014 (Figure 1). Our study also identify 7 cases Candida infection before education program dan 
8 cases after education program.

Based on the result of antibiotic susceptibility patterns pre-education step, Cefoperazone/Sulbactam and Amikacin were used as empirical therapy (Table 2). Even though Amikacin sensitivity was only $21.4 \%$, this antibiotic was still used as combination therapy for Acinetobacter baumanii, Pseudomonas aeruginosa and Klebsiela pneumonia. We used local antibiotic usage guideline developed from the existing published guidelines and the result of antibiotic susceptibility testing post-education to assess the appropriateness of antibiotic use.

Almost all of Acinetobacter baumanii isolate in this study were multi-drug resistant. The antibiotic susceptibility rate of Acinetobacter baumanii to 9 antibiotic regimens is highlighted in Table 3 . The outcome of a com-

Table 1. Type of specimen for culture examination during pre-education and post-education.

\begin{tabular}{ccc}
\hline Type of specimen & Pre-education $(\mathrm{n}=39)$ & Post-education $(\mathrm{n}=62)$ \\
Urine & $0(0.0 \%)$ & $2(3.2 \%)$ \\
Sputum & $33(84.5 \%)$ & $28(45.2 \%)$ \\
LCS & $0(0.0 \%)$ & $1(1.6 \%)$ \\
Blood & $5(13 \%)$ & $31(50 \%)$ \\
Bronchial Secretion & $1(2.5 \%)$ & $0(0.0 \%)$ \\
\hline
\end{tabular}

Table 2. Antibiotic use for empirical therapy ${ }^{\mathrm{a}}$.

\begin{tabular}{|c|c|c|}
\hline \multirow{2}{*}{ Pathogen isolate } & Combination therapy & Adult dosage \\
\hline & Cefoperazone/Sulbactam & Cefoperazone:Sulbactam ratio $=1: 1$ \\
\hline Acinetobacter baumanii & & Mild infection: 1 - 2 gr/IV every 12 hours \\
\hline \multirow[t]{2}{*}{ Pseudomonas aeruginosa } & plus & Severe infection: max $4 \mathrm{gr} / \mathrm{IV} /$ day every 12 hours \\
\hline & & $\mathrm{CrCl}<30 \mathrm{ml} / \mathrm{min}:$ Sulbactam $2 \mathrm{gr} / \mathrm{IV} /$ day \\
\hline \multirow[t]{3}{*}{ Klebsiella pneumoniae } & Amikacin & $\mathrm{CrCl}<15 \mathrm{ml} / \mathrm{min}:$ Sulbactam $1 \mathrm{gr} / \mathrm{IV} /$ day \\
\hline & & 15 - 20 mg/kg/IV every 24 day \\
\hline & & 20 - $45 \mathrm{mg} / \mathrm{kg} / \mathrm{IV}$ every 6 - 12 day \\
\hline \multirow[t]{2}{*}{ Staphylococcus spp. } & Vancomycin & $\mathrm{CrCl}<30 \mathrm{ml} / \mathrm{min}: 10-15 \mathrm{mg} / \mathrm{kg}$ every $24 \mathrm{jam}$ \\
\hline & & $\mathrm{CrCl}<15 \mathrm{ml} / \mathrm{min}: 10-15 \mathrm{mg} / \mathrm{kg}$ every $48 \mathrm{jam}$ \\
\hline
\end{tabular}

${ }^{\mathrm{a}}$ Adapted from local antibiotic guideline.

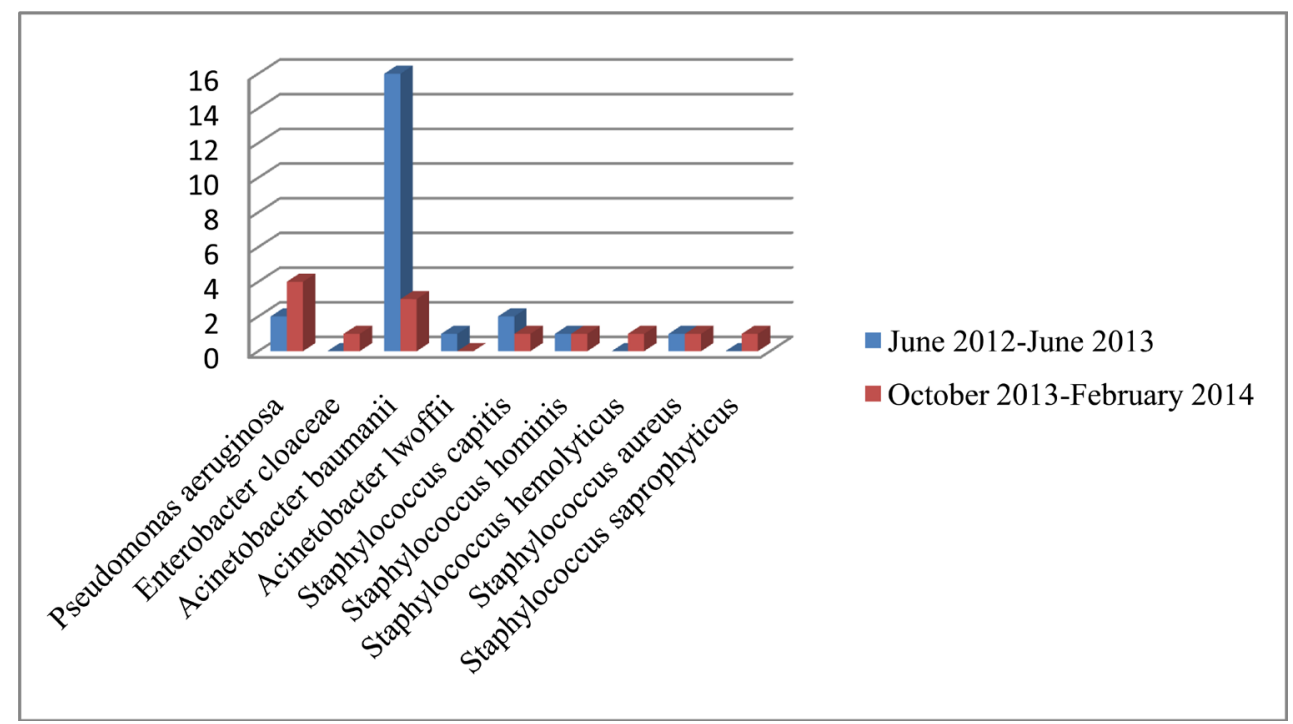

Figure 1. Number of Acinetobacter baumanii in June 2012-June 2013 (pre-education) and October 2013-February 2014 (post-education). 
Table 3. Antibiotic susceptibility rate of Acinetobacter baumanii, June 2012-June 2013 (pre-education) and October 2013February 2014 (post-education).

\begin{tabular}{ccc}
\hline Antibiotics & Pre-education (\%) & Post-education (\%) \\
\hline Piperacillin/tazobactam & 5 & 89 \\
Cefoperazone/sulbactam & 77 & 89 \\
Ceftazidime & 5 & 67 \\
Cefepime & 10 & 17 \\
Imipenem & 16 & 89 \\
Meropenem & 41.6 & 89 \\
Amikacin & 21 & 100 \\
Levofloxacin & 18 & 67 \\
Tigecycline & 35.3 & 57 \\
\hline
\end{tabular}

bination between education and appropriate use of antibiotics had elicited significant shrinkage of MDR-A. baumanii cases compare with non-A. baumanii from 70.8\% (17/24) in June 2012-June 2013 to 23\% (3/13) cases in October 2013-March 2014; P-value 0.006.

Education and appropriate use of antibiotics has become a main factor to help prevent the transmission of MDR-A. baumanii in ICU in Indonesia where the study conducted (OR 6.4; 95\% CI 1.56 - 26.30). Table 3 showed significant increase of antibiotic susceptibility rate in every antibiotic such as Piperacillin/Tazobactam (89\%), Cefoperazone/Sulbactam (89\%), Ceftazidime (67\%), Imipenem (89\%), Meropenem (89\%) and Amikacin $(100 \%)$.

\section{Discussion}

In this study, MDR-A. baumanii is defined as a strain that is resistant to three or more antibiotics such as anti pseudomonalpenicillins or $\beta$-lactam, anti pseudomonalcephalosporins, fluoroquinolone, carbapenems, and aminoglycoside [12] [13]. Recently, increasing number of MDR-gram negative cases are followed by increasing number of MDR-Acinetobacter baumanii hospital-acquired infections [7] [14] [15]. The decreased number of MDR-A. baumani cases in intensive care where this study was held was followed by increasing MDR-P. aeruginosa organisms. This finding proved that MDR-P. aeruginosa was the most commonly isolated organisms in intensive care patients, contributing 50\% (4/8) from all gram negative bacteria. The increasing uses of broad-spectrum antibiotics plays a significant role in associated this phenomenon. Even though the appropriate use of antibiotics therapy and de-escalation of therapy depending on culture and susceptibility testing have been enforced, large numbers of MDR-P. aeruginosa cases were still unsolved [12] [16].

MDR-A. baumanii is one of many important pathogens causing hospital acquired infection [6] [13] [15]. In this study, all of $A$. baumanii mainly were isolated from lower respiratory tract. These findings are similar with other result where $A$. baumanii were found in $27 \%$ patients [17]. Carbapenem resistant $A$. baumanii has endemically emerged globally including in Indonesia [18]. All of isolates before the study was initiated showed that there were no appropriate empirical antibiotic therapies. Aminoglycosides such as Amikacin were not so useful anymore since its sensitivity rate only $21 \%$. This finding is supported by Dent et al. [19] who found as much as 58\% isolates of A. baumnaii are resistant to Imipenem, Amikacin, and Ampicillin-Sulbactam, also similar with study conducted in Turkey that only Amikacin was still sensitive [17]. On the other hand, this problem comparable with increased in appropriate use of Carbapenems and Ciprofloxacin in hospitals [20]-[22].

Appropriate antibiotic use practices still become a challenge with various issue. Several approaches should be implemented before and after prescribing antibiotics such as restricted formulary, comply with local hospital antibiotic guideline, communicate with clinical pharmacologist and clinical microbiologist [23] [24]. Transmission of hospital acquired infection mainly via hands of health care workers, but the compliance of hand hygiene still low around 50\%. A study conducted by Allegranzi et al. in 2009 showed significant improvement of hand hygiene knowledge and compliance after educational session [25]. Therefore, hand hygiene education is one of the strategies that can be used to prevent the spread of infection in hospitals [10].

The limitation of our observation could not measure the compliance of recommended hand hygiene practice 
which may affect the incidence of health care associated infection in our intensive care. The number of positive culture in our study population was of small sample size and specific for intensive care setting, therefore not representative for all other hospital settings.

\section{Conclusion}

Our study found out that education improving around hand hygiene, appropriate antibiotic prescribing following local hospital guideline and the result of antibiotic sensitivity has been shown to reduce the transmission of MDR-A. baumanii in unit in within the Indonesian hospital. This approach was quite easy to apply in developing countries, such as Indonesia.

\section{Acknowledgements}

We are grateful to patients, nurses, and administrative staff of the intensive care unit who made this work possible. We also like thank to the laboratory for generous help and support throughout the study.

\section{Conflict of Interests}

The authors declare no conflict of interests.

\section{Funding}

This study was supported by Health Professional Education Quality (HPEQ) Project from International Bank for Reconstruction and Development (IBRD) (Loan No. 77370-ID).

\section{References}

[1] Kyungwon, L., Dongeun, Y., Seok-Hoon, J. and Yunsop, C. (2011) Multidrug-Resistant Acinetobacter spp.: Increasingly Problematic Nosocomial Pathogens. Yonsei Medical Journal, 52, 879-891. http://dx.doi.org/10.3349/ymj.2011.52.6.879

[2] Perween, N., Sehgal, S. and Prakash, K. (2014) Geographical Patterns in Antimicrobial Resistance of Acinetobacter in Clinical Isolates. Journal of Clinical and Diagnostic Research, 8, DC10-DC12.

[3] Wisplinghoff, H., Bischoff, T., Tallent, S.M., Seifert, H., Wenzel, R.P. and Edmond, M.B. (2004) Nosocomial Bloodstream Infections in US Hospitals: Analysis of 24,178 Cases from a Prospective Nation Wide Surveillance Study. Clinical Infectious Diseases, 39, 309-317. http://dx.doi.org/10.1086/421946

[4] Rungruanghiranya, S., Somboonwit, C. and Kanchanapoom, T. (2005) Acinetobacter Infection in the Intensive Care Unit. Journal of Infectious Diseases and Antimicrobial Agents, 22, 77-88.

[5] Cerqueira, G.M. and Peleg, A.Y. (2011) Insights into Acinetobacter baumannii Pathogenicity, IUBMB Life, 63, 10551060. http://dx.doi.org/10.1002/iub.533

[6] Peleg, A.Y., de Breij, A., Adams, M,D., Cerqueira, G.M., Mocali, S., Galardini, M., et al. (2012) The Success of Acinetobacter Species; Genetic, Metabolic, and Virulence Attributes. PLoS ONE, 7, e46984.

[7] Perez, F., Hujer, A.M., Hujer, K.M., Decker, B.K., Rather, P.N. and Bonomo, R.A. (2007) Global Challenge of Multidrug-Resistant Acinetobacter baumannii. Antimicrobial Agents and Chemotherapy, 51, 3471-3484. http://dx.doi.org/10.1128/AAC.01464-06

[8] Fournier, P.E. and Richet, H. (2006) The Epidemiology and Control of Acinetobacter baumannii in Healthcare Facilities. Clinical Infectious Disease, 42, 692-699. http://dx.doi.org/10.1086/500202

[9] Sureshg, J., Geetanjalim, L., Meenakshig, S., Nilimav, T., Vikrams, G. and Krishnab, N. (2006) Clinical and Demographic Features of Infection Cased by Acinetobacter Species. Indian Journal of Medical Sciences, 60, 351-360. http://dx.doi.org/10.4103/0019-5359.27219

[10] Allegranzi, B., Bagheri, N.S., Combescure, C., Graafmans, W., Attar, H., Donaldson, L., et al. (2011) Burden of Endemic Health-Care-Associated Infection in Developing Countries: Systematic Review and Meta-Analysis. The Lancet, 377, 228-241. http://dx.doi.org/10.1016/S0140-6736(10)61458-4

[11] Clinical and Laboratory Standard Institute (2012) Performance Standards for Antimicrobial Susceptibility Testing, Twenty-Second Informational Supplement.

[12] Kunz, A.N. and Brook, I. (2010) Emerging Resistant Gram-Negative Aerobic Bacilli in Hospital-Aquired Infections. Chemotherapy, 56, 492-500. http://dx.doi.org/10.1159/000321018 
[13] Cisneros, J.M. and Rodriguez-Bano, J. (2002) Nosocomial Bacteremia Due to Acinetobacter baumannii: Epidemiology, Elinical Features, and Treatment. Clinical Microbiology and Infection, 8, 687-693. http://dx.doi.org/10.1046/j.1469-0691.2002.00487.x

[14] Howard, A., Donoghue, M.O., Feeney, A. and Sleator, R.D. (2012) Acinetobacter baumannii: An Emerging Opportunistic Pathogen. Virulence, 3, 243-250. http://dx.doi.org/10.4161/viru.19700

[15] Dijkshoorn, L., Nemec, A. and Harald, S. (2007) An Increasing Threat in Hospitals: Multidrug-Resistant Acinetobacter baumannii. Nature Reviews Microbiology, 5, 939-949. http://dx.doi.org/10.1038/nrmicro1789

[16] Mortensen, B.L. and Skaar, E.P. (2012) Host-Microbe Interactions That Shape the Pathogenesis of Acinetobacter baumannii Infection. Cellular Microbiology, 14, 1336-1344. http://dx.doi.org/10.1111/j.1462-5822.2012.01817.x

[17] Gonlugur, U., Bakici, M.Z., Akkurt, I. and Efeoglu, T. (2004) Antibiotica Susceptibility Patterns among Respiratory Isolates of Gram-Negative Bacilli in a Turkish University Hospital. BMC Microbiology, 4, 32. http://dx.doi.org/10.1186/1471-2180-4-32

[18] Weisenberg, S.A., Schuetz, A.N., Alexander, E.A., Eiss, B., Behta, M., et al. (2011) Endemic Acinetobacter baumanii in a New York Hospital. PLoS ONE, 6, e28566. http://dx.doi.org/10.1371/journal.pone.0028566

[19] Dent, L.L., Marshal, D.R., Pratap, S. and Hulette, R.B. (2010) Multidrug Resistant Acinetobacter baumanii: A Descriptive Study in a City Hospital. BMC Infectious Diseases, 10, 196. http://dx.doi.org/10.1186/1471-2334-10-196

[20] Khan, M.A., Mahomed, M.F., Ashshi, A.M. and Faiz, A. (2012) Drug Resistance Patterns of Acinetobacter baumanii in Makkah, Saudi Arabia. Pakistan Journal of Medical Research, 51, 127-131.

[21] Hsueh, P.R., Teng, L.J., Chen, C.Y., Chen, W.H., Yu, C.J., et al. (2002) Pandrug-Resistant Acinetobacter baumanii Causing Nosocomial Infections in a University Hospital, Taiwan. Emerging Infectious Diseases, 8, 827-832. http://dx.doi.org/10.3201/eid0805.020014

[22] Maragakis, L.L. and Perl, T.M. (2008) Acinetobacter baumannii: Epidemiology, Antimicrobial Resistance, and Treatment Options. Clinical Infectious Diseases, 46, 1254-1263. http://dx.doi.org/10.1086/529198

[23] Thuong, M., Shortgen, F., Zazempa, V., Girou, E., Soussy, C.J. and Brun-Buisson, C. (2000) Appropriate Use of Restricted Antimicrobial Agents in Hospitals the Importance of Empirical Therapy and Assisted Re-evaluation. Journal of Antimicrobial Chemotherapy, 46, 501-508. http://dx.doi.org/10.1093/jac/46.3.501

[24] Goldman, D.A., Weinstein, R.A., Wenzel, R.P., Tablan, O.C., Duma, R.J., et al. (1996) Strategies to Prevent and Control the Emergence and Spread Antimicrobial-Resistant Microorganisms in Hospitals: A Challenge to Hospital Leadership. JAMA, 275, 234-240. http://dx.doi.org/10.1001/jama.1996.03530270074035

[25] Allegranzi, B. and Pittet, D. (2009) Role of Hand Hygiene in Health-Care-Associated Infection Prevention. The Journal of Hospital Infection, 73, 305-315. http://dx.doi.org/10.1016/j.jhin.2009.04.019 